\title{
ICAM-1 mediates lung leukocyte recruitment but not pulmonary fibrosis in a murine model of bleomycin-induced lung injury
}

\author{
T. Matsuse*, S. Teramoto*, H. Katayama*, E. Sudo*, H. Ekimoto ${ }^{+}$, H. Mitsuhashi ${ }^{\star}$, Y. Uejima ${ }^{\star}$, Y. \\ Fukuchi* $^{*}$ Y. Ouchi*
}

ICAM-1 mediates lung leukocyte recruitment but not pulmonary fibrosis in a murine model of bleomycin-induced lung injury. T. Matsuse, S. Teramoto, H. Katayama, E. Sudo, H. Ekimoto, H. Mitsuhashi, Y. Uejima, Y. Fukuchi, Y. Ouchi. (C) ERS Journals Ltd 1999.

ABSTRACT: Bleomycin-induced lung injury has been extensively used as a model of interstitial pneumonia and pulmonary fibrosis. Intercellular adhesion molecule (ICAM)-1 is a ligand for lymphocyte function-associated antigen (LFA)-1 $\alpha$ and has been shown to be required for leukocyte migration into inflamed areas. The purpose of this report was to investigate the role of the ICAM-1/LFA-1 $\alpha$ pathway in a murine model of bleomycin-induced lung injury.

Animals received $75 \mathrm{mg} \cdot \mathrm{kg}^{-1}$ bleomycin (BLM) i.v. followed by treatment with phosphate-buffered saline (BLM group), anti-ICAM-1 and LFA-1 $\alpha$ monoclonal lavage (BAL) fluid, hydroxyproline content and histological findings were compared between these groups.

In the BLM group, significant increases in total cell count, macrophage count and neutrophil count of BAL fluid were observed on days 7 and 14. In the BLM+mAb group, bleomycin-induced accumulation of neutrophils was significantly reduced on days 7 and $14(p<0.01)$. However, the administration of mAb to ICAM-1 and LFA-1 $\alpha$ did not decrease the lung hydroxyproline content or the histopathological fibrosis grading score, indicating that the antagonism of ICAM-1 and LFA-1 $\alpha$ did not attenuate bleomycin-induced pulmonary fibrosis.

This study suggests that the intercellular adhesion molecule-1/lymphocye functionassociated antigen-1 $\alpha$ pathway mediates the accumulation of inflammatory cells in the injured lung caused by bleomycin; however, other mechanisms are important for the subsequent development of pulmonary fibrosis.

Eur Respir J 1999; 13: 71-77. antibodies (mAb) (BLM+mAb group). Inflammatory cell counts of bronchoalveolar

*Dept of Geriatrics, Faculty of Medicine, University of Tokyo, Tokyo, Japan ${ }^{+}$Anticancer Drugs Dept, Pharmaceutical Group, Nippon Kayaku Co. Ltd, Tokyo, Japan. ${ }^{*}$ Teijin Institute for Bio-Medical Research, Tokyo, Japan.

Correspondence: T. Matsuse

Dept of Geriatrics

Faculty of Medicine

University of Tokyo

7-3-1 Hongo, Bunkyo-ku

Tokyo 113

Japan

Fax: 81358006530

Keywords: Adhesion molecules bleomycin

intercellular adhesion molecule-1

lymphocyte function-associated antigen$1 \alpha$

pulmonary fibrosis

Received: November 201998 Accepted after revision September 141998

Supported in part by a grant from the Ministry of Education, Science and Culture, Japan (grant no. 07670660), the Smoking Research Foundation and the Naito Foundation.

Pulmonary fibrosis is characterized by the presence of chronic inflammation and increased deposition of collagen in lung parenchyma $[1,2]$. Bleomycin is a well-known toxic substance which produces lung injury and pulmonary fibrosis in both humans and other animals. Bleomycininduced lung injury has been extensively used as a model of interstitial pneumonia and pulmonary fibrosis [3-6]. Many types of cell, such as macrophages, neutrophils and lymphocytes, are potential participants in the inflammatory process of pulmonary fibrosis. In particular, macrophages recruited to the lung are generally supposed to be involved in the evolution of pulmonary fibrosis by the secretion of fibrogenic cytokines $[1,4,5,7]$, whereas the neutrophils accumulated in the lung are supposed to play some role in ameliorating fibrogenesis $[3,8]$.

In the process ofleukocyte migration into inflamed tissue, it is essential for leukocytes to adhere to the microvascular endothelium [9]. Intercellular adhesion molecule (ICAM)1 is a member of the immunoglobulin (Ig) superfamily and a ligand for lymphocyte function-associated antigen (LFA)$1 \alpha[10]$. It has been reported that ICAM-1 is required for leukocyte migration into inflamed areas [11-13] and plays an important role in inflammatory pulmonary disease, in- cluding bronchial asthma and hyperoxia-induced damage [14-16]. The purpose of this report was to investigate the role of the ICAM-1/LFA-1 $\alpha$ pathway in a murine model of lung injury and the subsequent development of pulmonary fibrosis induced by bleomycin.

\section{Materials and methods}

\section{Monoclonal antibodies}

A rat anti-mouse ICAM-1 monoclonal antibody (mAb; clone KAT-1, rat IgG2a) [17] and a rat anti-mouse LFA-1 $\alpha$ (CD11a) mAb (clone KBA, rat IgG2a) [18, 19] were purchased from Seikagaku Co. (Tokyo, Japan). A control polyclonal rat IgG was purchased from Organo Teknika Corporation (Durham, NC, USA). These $\mathrm{mAb}$ and IgG were free from azide and other preservatives.

\section{Preparation of animals}

Seventy-one male 8-week-old Institute for Cancer Research (ICR) mice (Japan S.L.C., Shizuoka, Japan), weighing 33-35 g, were studied and maintained in a specific 
pathogen-free environment. Mice were divided into four experimental groups: 1) saline-i.v.-challenged, phosphatebuffered saline (PBS)-treated group (control (CTRL), group, $\mathrm{n}=27)$; 2) bleomycin (BLM)-i.v.-challenged, PBStreated group (BLM group, $\mathrm{n}=22$ ); 3) bleomycin-i.v.challenged, anti-ICAM-1 and LFA- $1 \alpha$ mAb-treated group $(\mathrm{BLM}+\mathrm{mAb}$ group, $\mathrm{n}=21)$; and 4) bleomycin-i.v.-challenged, control IgG-treated group (BLM+IgG group, $\mathrm{n}=21$ ). The mice in the BLM, BLM $+\mathrm{mAb}$ and $\mathrm{BLM}+\mathrm{IgG}$ groups received i.v. $75 \mathrm{mg} \cdot \mathrm{kg}^{-1}$ of bleomycin (Nippon Kayaku Co., Tokyo, Japan). The mice in the CTRL group received i.v. the same volume of saline $(0.15 \mathrm{~mL})$. Immediately after bleomycin or saline injection, animals in the CTRL and BLM groups were treated i.v. with $0.20 \mathrm{~mL}$ of PBS, followed by i.p. administration of $0.3 \mathrm{~mL}$ of PBS. The mice in the $\mathrm{BLM}+\mathrm{mAb}$ group were treated i.v. with $0.7 \mathrm{mg} \cdot \mathrm{kg}^{-1}$ of anti-mouse ICAM-1 mAb and $0.7 \mathrm{mg} \cdot \mathrm{kg}^{-1}$ of antimouse LFA- $1 \alpha \mathrm{mAb}$, followed by i.p. administration of $1.0 \mathrm{mg} \cdot \mathrm{kg}^{-1}$ of anti-mouse ICAM-1 mAb and 1.0 $\mathrm{mg} \cdot \mathrm{kg}^{-1}$ of anti-mouse LFA- $1 \alpha \mathrm{mAb}$. The mice in the $\mathrm{BLM}+\mathrm{IgG}$ group were treated i.e. with $1.4 \mathrm{mg} \cdot \mathrm{kg}^{-1}$ of control rat IgG, followed by i.p. administration of $2.0 \mathrm{mg} \cdot \mathrm{kg}^{-1}$ of control rat IgG. At days 3 and 5 after bleomycin or saline administration, animals in each group received i.p. the same amount of PBS, anti-mouse ICAM-1 and antimouse LFA- $1 \alpha \mathrm{mAb}$ and control IgG, respectively.

\section{Bronchoalveolar lavage fluid and assessment of differ- ential cell count}

On days 7 and 14 after $i . v$. bleomycin administration, bronchoalveolar lavage (BAL) was performed $(0.7 \mathrm{~mL}$ saline administered three times) in five animals from each group. After the BAL fluid was centrifuged, the total and differential cell counts of the BAL fluid were determined from the cell fraction.

\section{Lung hydroxyproline contents}

To estimate the total lung collagen content, the hydroxyproline content was measured according to an established method with modifications in five or 11 animals in each group on day 14 [20]. In brief, the whole lung from each animal was dissected free of the major bronchi. The lobes were homogenized and then hydrolysed in $6 \mathrm{~N} \mathrm{HCl}$ for $24 \mathrm{~h}$ at $110^{\circ} \mathrm{C}$. After centrifugation, the hydrolysate was neutralized with $\mathrm{KOH}$. Each $0.25 \mathrm{~mL}$ of sample was mixed with $0.25 \mathrm{~mL}$ of $0.01 \mathrm{M} \mathrm{CuSO}_{4}, 0.25 \mathrm{~mL}$ of $2 \mathrm{~N} \mathrm{NaOH}$ and $0.25 \mathrm{~mL}$ of $6 \% \mathrm{H}_{2} \mathrm{O}_{2}$. Then, each sample was analysed colorimetrically for hydroxyproline content after the addition of $1 \mathrm{~mL}$ of $1.5 \mathrm{~N} \mathrm{H}_{2} \mathrm{SO}_{4}$ and $0.5 \mathrm{~mL}$ of $5 \%$ dimethylaminobenzaldehyde. The hydroxyproline concentration of each sample was determined from the standard curve giving the absorbance at $560 \mathrm{~nm}$ by the known concentrations of reagent hydroxyproline.

\section{Histopathology}

Thirty-five days after bleomycin or saline administration, six animals from each group were killed by cervical dislocation followed by exsanguination. Midsagittal 5- $\mu \mathrm{m}$ thick sections of paraffin-embedded lung tissues were prepared and stained with Azan. The severity of pulmonary fibrosis in these sections was scored semiquantitatively according to the method of AsHCROFT et al. [21], with minor modifications [22], as follows. Grade 1: normal lung; grade 2: minimal fibrotic thickening of alveolar or bronchial walls; grade 3: moderate thickening of walls without obvious damage to lung architecture; grade 4: increased fibrosis with definite damage to lung structure and formation of fibrous bands or small fibrous masses; and grade 5: severe distortion of structure and large fibrous areas. The grading was performed in a double-blind fashion by examining more than 10 fields selected at random in each section.

\section{Data analysis}

Parametric comparisons of data among the four experimental groups were carried out with analysis of variance (ANOVA) in conjunction with Fisher's least squares difference (LSD) test. In a histopathological study, nonparametric comparison were made by the Kruskal-Wallis test in conjunction with the Scheffé multiple-comparisons t-test. Data were expressed as mean \pm SEM. A p-value $<0.05$ was taken as significant.

\section{Results}

Bronchoalveolar lavage fluid and assessment of differential cell count

The recovery rates of BAL and total cell count of BAL fluid on days 7 and 14 after bleomycin treatment are shown in tables 1 and 2. Macrophages, lymphocyte and neutrophil counts in each experimental group on days 7 and 14 are shown in figures 1 and 2 , respectively. There were no significant differences in the recovery rate of BAL among the four groups (tables 1 and 2). The number of macrophages, lymphocytes and neutrophils in the BLM group

Table 1. - Bronchoalveolar lavage on day 7

\begin{tabular}{lcccc}
\hline & CTRL & BLM & BLM+mAb & BLM+IgG \\
\hline Recovery rate \% & $84.4 \pm 2.7$ & $85.9 \pm 3.3$ & $84.5 \pm 3.5$ & $90.2 \pm 2.1$ \\
(range) & $(77.6-90.5)$ & $(76.2-95.2)$ & $(71.4-90.5)$ & $(85.7-95.2)$ \\
Total cell count $\times 10^{4}$ & $4.7 \pm 0.9$ & $74.3 \pm 21.4^{* *}$ & $23.6 \pm 3.0^{\dagger}$ & $26.8 \pm 5.2^{\dagger}$ \\
\hline
\end{tabular}

Values are means \pm SEM. CTRL: saline-challenged, phosphate-buffered saline (PBS)-treated group; BLM: bleomycin-challenged, PBStreated group; BLM $+\mathrm{mAb}$ : bleomycin-challenged, anti-intercellular adhesion molecule-1 and lymphocyte function-associated antigen$1 \alpha$ monoclonal antibody-treated group; BLM+IgG: bleomycin-challenged, control immunoglobulin G-treated group. There were five animals in each group. ${ }^{* *}: \mathrm{p}<0.01$ versus $\mathrm{CTRL}$ group; ${ }^{\dagger}: \mathrm{p}<0.01$ versus $\mathrm{BLM}$ group. 
Table 2. - Bronchoalveolar lavage on day 14

\begin{tabular}{lcccc}
\hline & CTRL & BLM & BLM $+\mathrm{mAb}$ & BLM+IgG \\
\hline Recovery rate \% & $84.5 \pm 1.7$ & $87.3 \pm 1.6$ & $82.8 \pm 1.8$ & $88.1 \pm 2.5$ \\
(range) & $(79.0-88.1)$ & $(82.4-9.10)$ & $(78.6-87.1)$ & $(78.6-92.9)$ \\
Total cell count $\times 10^{4}$ & $5.8 \pm 1.4$ & $61.6 \pm 13.0^{* *}$ & $16.3 \pm 3.0^{\dagger}$ & $41.1 \pm 7.2^{* *}$ \\
\hline
\end{tabular}

Values are means \pm SEM. For definitions see footnote to table 1 . There were five animals in each group. ${ }^{* *}: \mathrm{p}<0.01$ versus $\mathrm{CTRL}$ group; ${ }^{\dagger}$ : $\mathrm{p}<0.01$ versus BLM group.

was significantly greater than in the CTRL group on days 7 and $14(\mathrm{p}<0.01)$.

Bleomycin-induced increases in total cell count, the number of macrophages and neutrophils were significantly attenuated by the treatment with anti-ICAM-1 and LFA- $1 \alpha$ $\mathrm{mAb}$ after bleomycin challenge on days 7 and 14 (tables 1 and 2, figs. 1 and 2). However, the treatment of control IgG did not decrease the number of neutrophils significantly at either time point. Both the treatment with anti-ICAM-1 and LFA- $1 \alpha \mathrm{mAb}$ and that with control $\operatorname{IgG}$ decreased the number of lymphocytes on days 7 and 14 and the number of macrophages on day 7 .

\section{Hydroxyproline content of bleomycin-treated lungs}

Fourteen days after bleomycin treatment, total lung collagen, as measured by hydroxyproline content, increased in the BLM group $(69.9 \%$ increase, $p<0.01)$. Figure 3 shows the lung hydroxyproline content of each experimental group. The lung hydroxyproline content in both the BLM+ $\mathrm{mAb}$ group and the BLM+IgG group was not different from that in the BLM group.

\section{Histopathology}

There was no fibrotic lesion in tissue sections from control animals on day 14. In contrast, peripleural fibrosis was observed in both the BLM and BLM $+\mathrm{mAb}$ groups, as assessed by haematoxylin and eosin (H\&E) staining and Azan staining (fig. 4).

Photomicrographs of representative Azan staining of parenchymal tissue from the CTRL, BLM and BLM $+\mathrm{mAb}$ group animals on day 35 after bleomycin treatment are shown in figure 5 . Increased fibrosis with definite damage to the lung structure and formation of small fibrous masses was observed in the BLM and BLM $+\mathrm{mAb}$ group animals.

The fibrosis grading score in the BLM group on day 35 was significantly higher than that in the CTRL group ( $\mathrm{p}<$ 0.01 ) (fig. 6). However, there were no significant differences in fibrosis grading scores among the BLM, BLM+ $\mathrm{mAb}$ and $\mathrm{BLM}+\mathrm{IgG}$ groups. These results indicate that there were no significant inhibitory effects of anti-ICAM$1 / \mathrm{LFA}-1 \alpha \mathrm{mAb}$ on pulmonary fibrosis.

\section{Discussion}

Bleomycin stimulates a pulmonary inflammatory response, characterized by an increase in leukocyte infiltration, fibroblast proliferation and collagen deposition [23]. The results of the current study show that the ICAM-1/LFA- $1 \alpha$ pathway is involved in the pathogenesis of inflammation induced by bleomycin. Treatment with $\mathrm{mAb}$ to ICAM-1 and LFA- $1 \alpha$ in combination attenuated macrophage and neutrophil sequestration. Simultaneous administration of

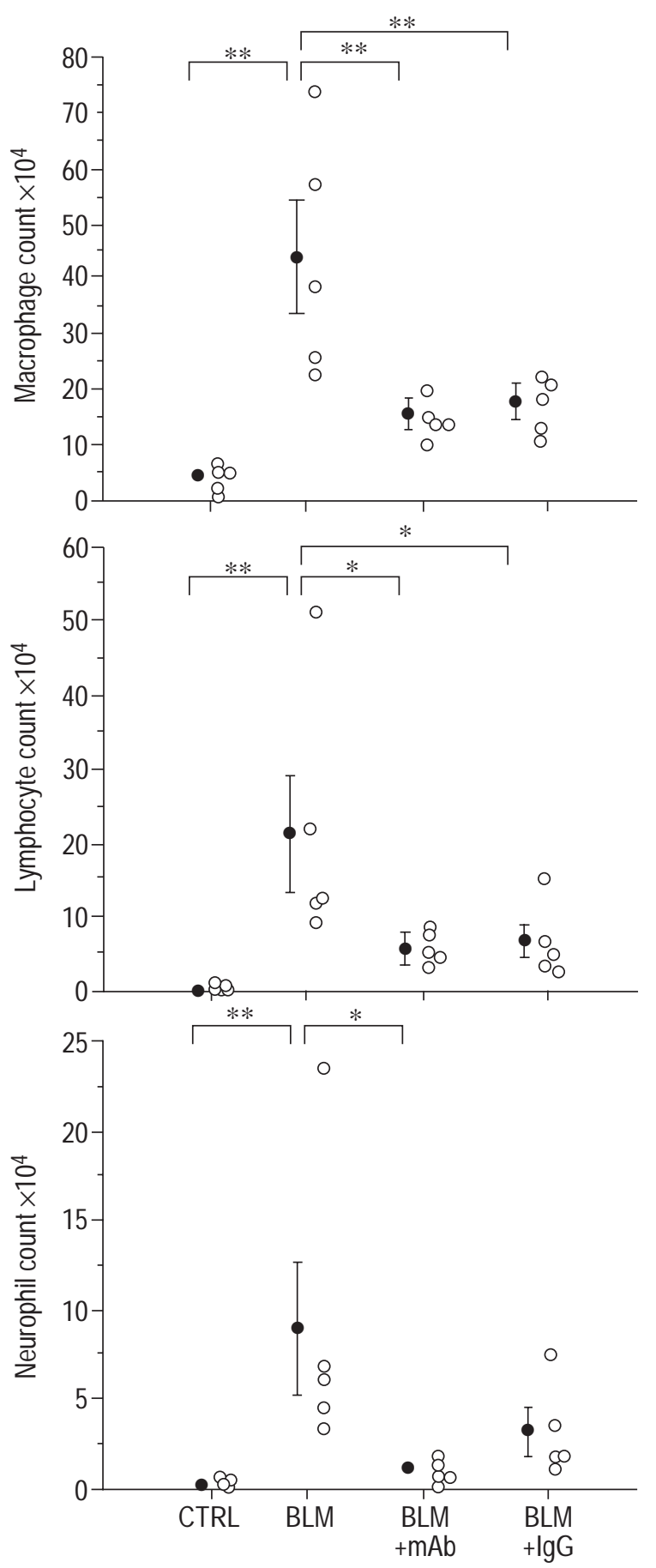

Fig. 1. - Macrophage, lymphocyte and neutrophil counts of bronchoalveolar lavage fluid in each experimental group: CTRL $(n=5)$, BLM $(n=5), B L M+m A b(n=5)$ and BLM $+\operatorname{IgG}$ group $(n=5)$ at day 7 . For definitions see footnote to table 1 . Values are presented as individual data $(\bigcirc)$ and group means $\pm \operatorname{SEM}(\bullet) . *$ : $\mathrm{p}<0.05 ; * *$ : $<<0.01$. 

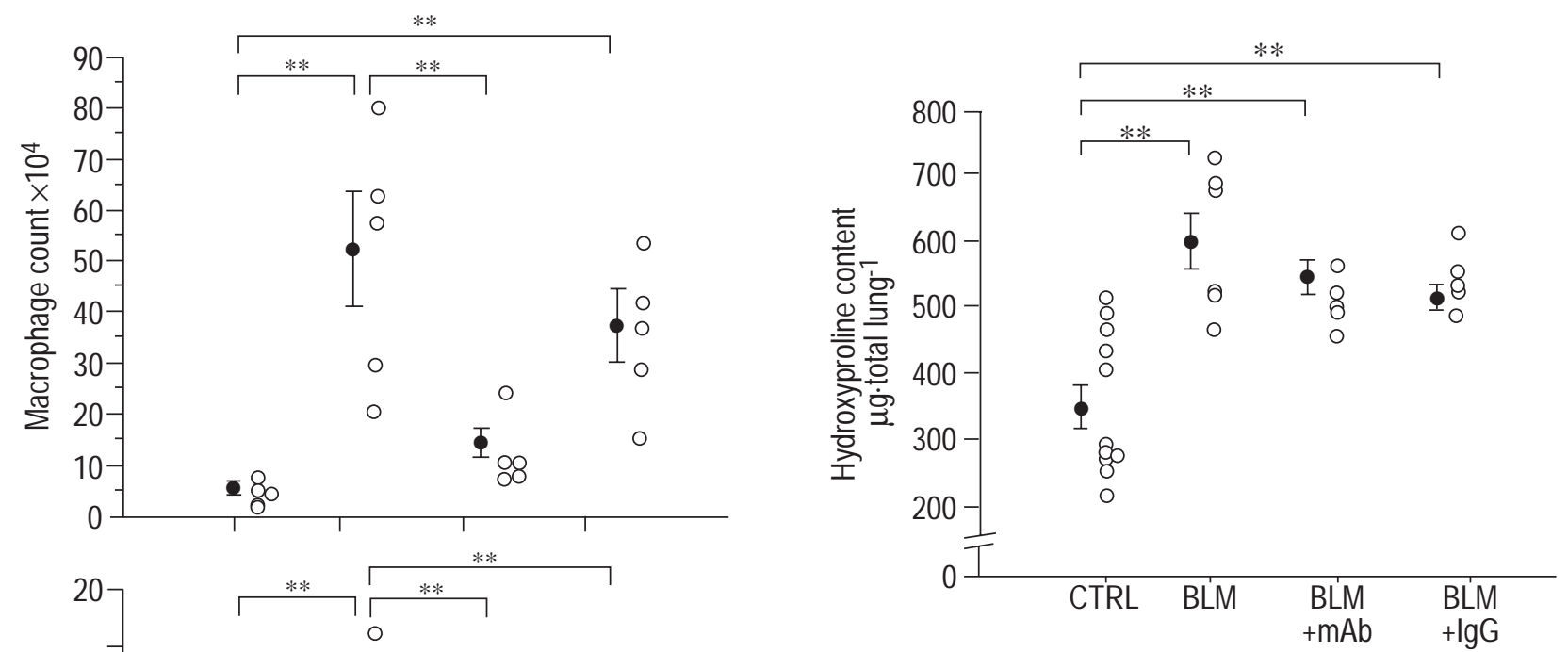

Fig. 3. - Lung hydroxyproline content of: CTRL $(n=10)$, BLM $(n=6)$, $\mathrm{BLM}+\mathrm{mAb}(\mathrm{n}=5)$ and BLM+IgG group $(\mathrm{n}=5)$ at day 14 . For definitions see footnote to table 1 . Values are presented as individual data $(\bigcirc)$ and group means $\pm \operatorname{SEM}(\bullet) . * *: \mathrm{p}<0.01$ versus $\mathrm{CTRL}$ group.

[11] and plays an important role in various inflammatory diseases [14-17, 19]. In the present study, it was hypothesized that the ICAM-1/LFA-1 $\alpha$ pathway could be involved in the pathogenesis of bleomycin-induced lung inflammation. To test this hypothesis, the effects of $\mathrm{mAb}$ to ICAM-1 and LFA-1 $\alpha$ on bleomycin-induced chronic lung inflammation were investigated in mice.

The results of this study demonstrate that bleomycin caused significant increases in total cell, macrophage, lymphocyte and neutrophil counts in BAL fluid and that these increases were significantly reduced by the administration of $\mathrm{mAb}$ to ICAM-1 and LFA- $1 \alpha$. The lymphocyte count on days 7 and 14 and macrophage count on day 7 were also reduced by the administration of control IgG. These findings indicate the possibility of nonspecific effects of the antibody used in this study, which has Fc receptors, on the immune system [17]. Neutrophil accumulation was completely attenuated by treatment with mAb to ICAM-1 and LFA- $1 \alpha$ and there were no effects of control IgG on this neutrophil accumulation on day 14. These findings suggest that these mAb to ICAM-1 and LFA- $1 \alpha$ specifically attenuate the neutrophil accumulation and that the ICAM-1/LFA- $1 \alpha$ pathway may be involved in the pathophysiological mechanism of neutrophil accumulation in bleomycin-induced lung inflammation in this model.

In a hamster model of pulmonary fibrosis caused by int-

Fig. 2. - Macrophages, lymphocyte and neutrophil counts of bronchoalveolar lavage fluid in each experimental group: CTRL $(n=5)$, BLM $(n=5), B L M+m A b(n=5)$ and BLM+IgG group $(n=5)$ at day 14 . For definitions see footnote to table 1 . Values are presented as individual data $(\bigcirc)$ and group means $\pm \operatorname{SEM}(\bullet)$. **: $\mathrm{p}<0.01$.

anti-ICAM-1 and LFA-1 $\alpha$ mAb completely reduced the neutrophil count to almost baseline levels. However, it did not inhibit bleomycin-induced pulmonary fibrosis.

In the process of leukocyte migration into inflamed areas, it is essential for leukocytes to adhere to the microvascular endothelium [9]. ICAM-1 is an $80-110 \mathrm{kDa}$ glycoprotein consisting of five immunoglobulin-like domains and a ligand for LFA-1 $\alpha[9,10]$. It has been demonstrated that the ICAM-1/LFA-1 $\alpha$ pathway functions in cell-cell adhesion reported that neutrophil elastase plays an important role in pulmonary fibrosis [24]. MituHASHi et al. [22] have also demonstrated that the administration of truncated secretory leukoprotease inhibitor ameliorates bleomycin-induced pulmonary fibrosis in hamsters. Thus, it is speculated that adhesion and activation of neutrophils might be the initial action, leading to the secretion of neutrophil elastase and increases in lung injury to develop pulmonary fibrosis. However, the role of neutrophils in pulmonary fibrosis is controversial. It is also supposed that increased neutrophils in pulmonary fibrosis may play some roles in ameliorating fibrogenesis because of increased collagenolytic activity and/or decreased collagen synthesis $[3,8]$. 

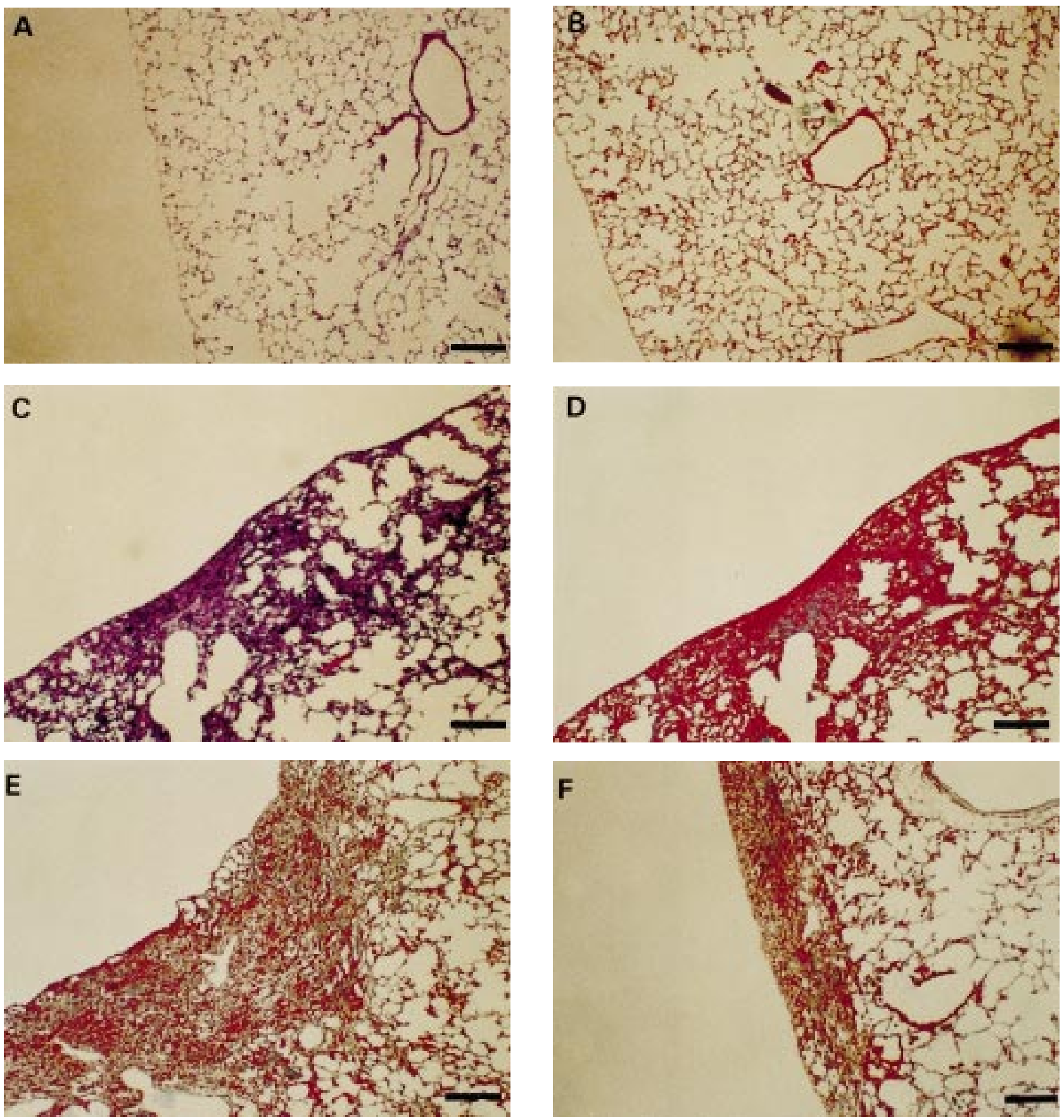

Fig. 4. - Photomicrographs of lung parenchymal tissues from: A) a saline-challenged animal treated with phosphate-buffered saline (PBS) (haematoxylin and eosin (H\&E) stain); B) a saline-challenged animal treated with PBS (the same tissue as in part A) (Azan stain); C) a bleomycin (BLM)-challenged animal treated with PBS (H\&E stain): D) a BLM-challenged animal treated with PBS (the same tissue as part C) (Azan stain); E) a BLM-challenged animal treated with anti-intercellular adhesion molecule-1 and lymphocyte function-associated antigen-1 $\alpha$ monoclonal antibodies (Azan stain); and F) a BLM-challenged animal treated with immunoglobulin G, at day 14 (Azan stain). (Internal scale bar $=80 \mu \mathrm{m}$.)

To test the role of the ICAM-1/LFA-1 $\alpha$-mediated neutrophil accumulation in pulmonary fibrosis induced by bleomycin, the effect of treatment with anti-ICAM-1/ LFA $-1 \alpha \mathrm{mAb}$ on histopathology and pulmonary collagen deposition was also studied. Fibrotic lesions were observed under the pleura in the BLM group on day 14. Moreover, a significant $69.9 \%$ increase in total collagen (hydroxyproline content) relative to control animals was observed in animals on day 14 . In addition to the increased lung hy- droxyproline content, the histopathological fibrosis grading score on day 35 in the BLM group was significantly higher than that in the control group.

Peripleural fibrosis was also observed in the BLM $+\mathrm{mAb}$ group. Furthermore, neither the lung collagen content on day 14 nor the histopathological fibrosis grading score on day 35 was significantly decreased by mAb to ICAM-1 and LFA- $1 \alpha$. These findings suggest that the ICAM-1/ LFA- $1 \alpha$ pathway does not play a pivotal role in the 

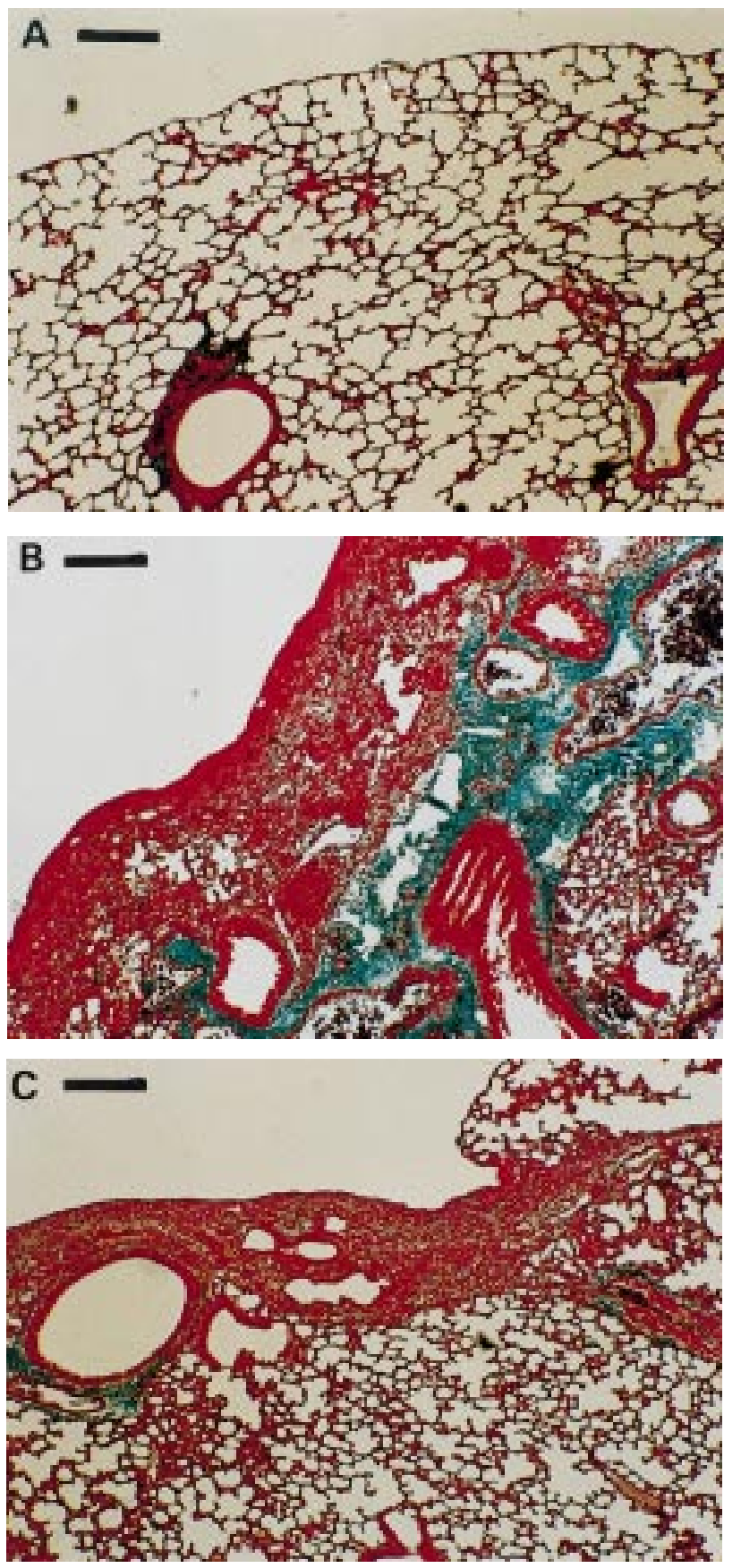

Fig. 5. - Photomicrographs of parenchymal tissues from: A) a salinechallenged animal treated with phosphate-buffered saline (PBS); B) a bleomycin (BLM)-challenged animal treated with PBS; and C) a BLMchallenged animal treated with anti-intercellular adhesion molecule-1 and lymphocyte function-associated antigen- $1 \alpha$ monoclonal antibodies, at day 35 (Azan stain). (Internal scale bars $=80 \mu \mathrm{m}$.)

subsequent development of pulmonary fibrosis induced by bleomycin. It is also suggested that the ICAM-1/LFA- $1 \alpha$ pathway-mediated neutrophil accumulation may have opposite effects on the fibrotic response to those of bleomycin. These findings indirectly support previous experimental studies in which neutrophil-depleted bleomycin-treated animals showed an increased lung collagen deposition and increased collagen synthesis $[3,8]$. In the present study, antagonism of ICAM-1 and LFA- $1 \alpha$ decreased the re-

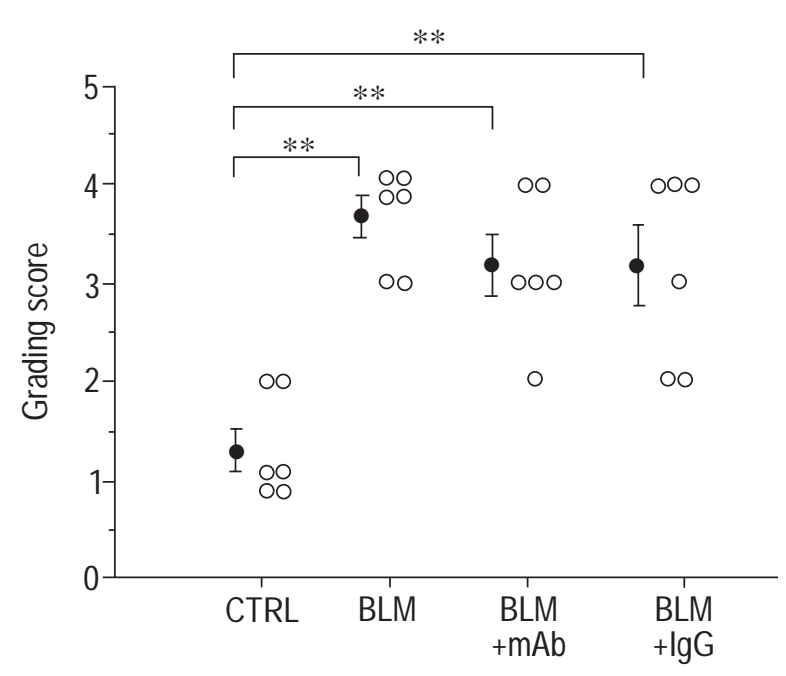

Fig. 6. - Histopathological changes at day 35 after bleomycin treatment in each experimental group: CTRL $(n=6)$, BLM $(n=6), B L M+m A b$ $(n=6)$ and BLM+IgG group $(n=6)$. The severity of lung fibrosis was score semiquantitatively according to the method of Ashcroft with minor modifications $[21,22]$. For definitions see footnote to table 1 . Values are presented as individual data $(\bigcirc)$ and group means $\pm \operatorname{SEM}(\bullet)$. **: $p<0.01$ versus CTRL group (analysis of variance in conjunction with Fisher's least squares difference test).

cruitment of macrophages, lymphocytes and neutrophils into the lungs of bleomycin-treated animals. While it has been supposed that the partially decreased levels of macrophages and lymphocytes may result in a partial decrease in lung collagen deposition, the complete decrease in neutrophil accumulation shown in this study may result in an increase in collagen synthesis and/or decrease in collagenolysis. Therefore, it would appear that these opposite effects may cancel each other out and that combined treatment of mAb to ICAM- 1 and LFA- $1 \alpha$ did not reduce the lung collagen deposition in this model.

The present data, however, seem to contradict those reported by PigueT and coworkers [25, 26], who showed that administration of an antibody to either CD-11a (LFA$1 \alpha$ ) or CD-11b caused inhibition of pulmonary fibrosis induced by bleomycin and silica and that this inhibition correlated with a reduction in platelet trapping in lung tissue [26]. Furthermore, they reported that treatment with an anti-CD11a or anti-CD11b antibody had little or no effect on the cellularity of BAL. These differences between the present data and those of PIGUET and coworkers [25, 26] may be related to either the dose or the potency of antibodies, or both. In particular, it is most likely that the failure to reduce the number of cells recovered by BAL in the previous study was due to the lack of simultaneous administration of the anti-ICAM-1 antibody. Because two mAb to ICAM- 1 and LFA- $1 \alpha$ are supposed to act synergistically [19], the administration of the mAb to CD11a (LFA-1 $\alpha$ ) alone may not be enough to reduce the number of cells in the BAL. The difference in the effect of antibodies on lung collagen deposition induced by bleomycin in the present data and those of PIGUET and coworkers [25, 26] may also be due to the extent of reduction of neutrophil recruitment. In contrast with their experimental model, wherein the number of neutrophils did not decrease with anti-CD11a $\mathrm{mAb}$ treatment, the number of neutrophils was reduced 
almost to the baseline value by mAb to ICAM-1 and LFA$1 \alpha$ in the present study, which may have promoted lung collagen deposition $[3,8]$.

SMiтh et al. [27] demonstrated that treatment with antimonocyte inflammatory protein (MIP)- $1 \alpha$ antibodies significantly reduced pulmonary mononuclear cell accumulation and fibrosis in bleomycin-challenged mice, which conflicts to some extent with the present findings. However, passive immunization with anti-MIP- $1 \alpha$ antibodies did not reduce pulmonary neutrophils [27]. Therefore, the difference between their findings and the present results could also be explained by the lack of a reduction in neutrophils in their study.

In summary, this study demonstrated that the antibodies to intercellular adhesion molecule-1 and lymphocyte function-associated antigen- $1 \alpha$ significantly attenuated bleomycin-induced recruitment of neutrophils into the lung. However, these antibodies had no effect on bleomycin-induced pulmonary fibrosis, despite the considerable inhibition of leukocyte recruitment. These observations suggest that the intercellular adhesion molecule-1/lymphocyte function-associated antigen- $1 \alpha$ pathway contributes significantly to the accumulation of neutrophils in the bleomycin-induced lung lesion, but is not a key step in the development of pulmonary fibrosis. It is also likely that neutrophils accumulating in the lung by the intercellular adhesion molecule-1/lymphocyte function-associated antigen- $1 \alpha$ pathway play a role in the attenuation of bleomycin-induced pulmonary fibrosis.

Acknowledgements. The authors would like to thank T. Oka, E. Ohga, T. Nagase and M. Eto for helpful assistance and advice. The authors are also grateful to J.P. Barron for reviewing the manuscript.

\section{References}

1. Crystal RG, Bitterman PB, Rennard SI, Hance AJ, Keogh BA. Interstitial lung diseases of unknown cause. Disorders characterized by chronic inflammation of the lower respiratory tract. N Engl J Med 1984; 310: 154-166.

2. Colby TV, Churg AC. Patterns of pulmonary fibrosis. Pathol Annu 1986; 21: 277-309.

3. Clark JG, Kulin C III. Bleomycin-induced pulmonary fibrosis in hamsters: effect of neutrophil depletion on lung collagen synthesis. Am Rev Respir Dis 1982; 126: 737-739.

4. Thrall RS, Barton RW, D'amato DA, Sulavik SB. Differential cellular analysis of bronchoalveolar lavage fluid obtained at various stages during the development of bleomycin-induced pulmonary fibrosis in the rat. Am Rev Respir Dis 1982; 126: 488-492.

5. Phan SH, Kunkel SL. Inhibition of bleomycin-induced pulmonary fibrosis by nordihydroguiaretic acid: the role of alveolar macrophage activation and mediator production. Am J Pathol 1986; 124: 343-352.

6. Matsuse T, Fukuchi Y, Eto Y, et al. Expression of immunoreactive and bioactive activin A protein in adult murine lung after bleomycin treatment. Am J Respir Cell Mol Biol 1995; 13: 17-24.

7. Kovacs EJ. Fibrogenic cytokines: the role of immune mediators in the development of scar tissue. Immunol Today 1991; 12: 17-23.

8. Trall RS, Phan SH, McCormick JR, Ward PA. The development of bleomycin induced pulmonary fibrosis in neutrophil-depleted and complement-depleted rats. $A m ~ J$ Pathol 1981; 105: 76-81.
9. Springer TA. Adhesion receptors of the immune system. Nature 1990; 346: 425-434.

10. Marlin SD, Springer TA. Purified intercellular adhesion molecule-1 (ICAM-1) is a ligand for lymphocyte function-associated antigen 1 (LFA-1). Cell 1987; 51: 813-819.

11. Boyd AW, Wawryk SO, Burns GF, Fecondo JV. Intercellular adhesion molecule-1 (ICAM-1) has a central role in cell-cell contact-mediated immune mechanisms. Proc Natl Acad Sci USA 1988; 85: 3095-3099.

12. Smith CW, Rothlein R, Hughes BJ, et al. Recognition of an endothelial determinant for CD18-dependent human neutrophil adherence and transendothelial migration. $J$ Clin Invest 1988; 82: 1746-1756.

13. Barton RW, Rothlein R, Ksiazek J, Kennedy C. The effect of anti-intercellular adhesion molecule-1 on phorbolester-induced rabbit lung inflammation. J Immunol 1989; 143: $1278-1282$.

14. Wegner CG, Gundel RH, Reilly P, Haynes N, Letts LG, Rothlein R. Intercellular adhesion molecule-1 (ICAM-1) in the pathogenesis of asthma. Science 1990; 247: 456-459.

15. Kang BH, Crapo JD, Wegner CD, Letts LG, Chang LY. Intercellular adhesion molecule-1 expression on the alveolar epithelium and its modification by hyperoxia. $\mathrm{Am} \mathrm{J}$ Respir Cell Mol Biol 1993; 9: 350-355.

16. Nagase T, Fukuchi Y, Matsuse T, Sudo E, Matsui H, Orimo H. Antagonism of ICAM-1 attenuates airway and tissue responses to antigen in sensitized rats. Am J Respir Crit Care Med 1995; 151: 1244-1249.

17. Seko Y, Matsuda H, Kato K, et al. Expression of intercellular adhesion molecule-1 in murine hearts with acute myocarditis caused by coxackievirus B3. J Clin Invest 1993; 91: 1327-1336.

18. Nishimura T, Yagi H, Yagita H, Uchiyama Y, Hashimoto Y. Lymphokine-activated cell-associated antigen involved in broad-reactive killer cell-mediated cytotoxity. Cell Immunol 1985; 94: 122-132.

19. Isobe M, Yagita H, Okumura E, Ihara A. Specific acceptance of cardiac allograft after treatment with antibodies to ICAM-1 and LFA-1. Science 1992; 225: 1125-1127.

20. Mlyada DS, Tappel AL. Colorimetric determination of hydroxyproline. Anal Chem 1956; 28: 909-910.

21. Ashcroft T, Simpson JM, Timbrell V. Simple method of estimating severity of pulmonary fibrosis on a numerical scale. J Clin Pathol 1988; 41: 467-470.

22. Mituhashi H, Asano S, Nonaka T, Hamamura I, Masuda K, Kiyoki M. Administration of truncated secretory leukoprotease inhibitor ameliorates bleomycin-induced pulmonary fibrosis in hamsters. Am J Respir Crit Care Med 1996; 153: 369-374.

23. Chandler DB. Possible mechanisms of bleomycin-induced fibrosis. Clin Chest Med 1990; 11: 21-30.

24. Nagai A, Aoshiba Y, Ishihara Y, et al. Administration of $\alpha$-protease inhibitor ameliorates bleomycin-induced pulmonary fibrosis in hamsters. Am Rev Respir Dis 1992; 145: 651-656.

25. Piguet PF, Rosen H, Vesin C, Grau GE. Effective treatment of the pulmonary fibrosis elicited in mice by bleomycin or silica with anti-CD-11 antibodies. Am Rev Respir Dis 1993; 147: 435-441.

26. Piguet PF, Vesin C. Pulmonary platelet trapping induced by bleomycin: correlation with fibrosis and involvement of the $\beta_{2}$ integrins. Int $J$ Exp Pathol 1994; 75: 321-328.

27. Smith RE, Strieter RM, Phan SH, et al. Production and function of murine macrophage inflammatory protein- $1 \alpha$ in bleomycin-induced lung injury. J Immunol 1994; 153: 4704-4712. 